\title{
Undivided attention improves postoperative anesthesia handover recall
}

This article was published in the following Dove Press journal:

Advances in Medical Education and Practice

10 July 2014

Number of times this article has been viewed

\section{Alejandro Arenas' \\ Burton J Tabaac' \\ Galina Fastovets ${ }^{2}$ \\ Vinod Patil ${ }^{3}$}

'Department of Clinical Sciences, American University of the Caribbean School of Medicine, Cupecoy, Sint Maarten; ${ }^{2}$ Department of Surgery, Broomfield Hospital, National Health Service, Chelmsford, UK; ${ }^{3}$ Department of Anesthesia, Queens Hospital, National Health Service, Romford, UK

Correspondence: Burton J Tabaac Department of Clinical Sciences, American University of the Caribbean School of Medicine, 508 Leon Circle, Langhorne, PA 19053, USA

Tel +I 2673344040

Email burton.tabaac@gmail.com
Background: For years, undivided attention during the presurgical "timeout" has been utilized as a precaution to ensure patient safety. The information relayed during the timeout is presented in a confirmatory nature rather than a delegation of new information. However, it is a standard of practice in which all members of the operating theater provide their full and undivided attention. Standards of patient care should be contiguous throughout the preoperative, perioperative, and postoperative stages of surgery. In this manner, it is expected that the same undivided attention afforded during the timeout should be maintained when transferring the patient to the postanesthesia care unit.

Methods: In this study, information was collected regarding handover of information during the transfer status postsurgical procedures. Data were collected via observing interactions between the anesthesiologist and the nurse during verbal patient transfers.

Results: This study demonstrated that the presence of undivided attention during the handover of a surgical patient in the postanesthesia care unit has a direct correlation with improved recall of the information discussed during handover.

Conclusion: Focus is on the quantity of information that can be recalled by the transferring nurse, and whether or not undivided attention affects the outcome. Analysis focuses on suggestions to better improve patient safety and recovery when being transferred in an anesthetic setting. The practice of patient handover should be standardized to better improve the safety and quality of medical care.

Keywords: communication, continuous quality improvement, healthcare quality improvement, safety culture, undivided attention, patient care

\section{Introduction}

Upon completion of a surgery in the operating room (OR), patients must be transferred, along with all pertinent information regarding their case, to the postanesthesia care unit (PACU). This information includes, but is not limited to, the patient's name, the procedure, medications given, and further directions or instructions for care. Patient information handover relies on the transfer of information, professional responsibility, and accountability between individuals and teams. ${ }^{1}$ Handover events carry inherent risk and depend greatly upon the teamwork and communication among health care professionals. ${ }^{2,3}$ Previously conducted studies have shown that lapses in proper handover protocol result in deleterious events. ${ }^{4-7}$ Patient information is transferred between staff from multiple professions in medicine with varying perspectives of the course of the surgery. Technical errors are prone to occur when there are communication failures between surgeons, anesthetists, and the anesthesia care team. ${ }^{6,8,9}$ 
The anesthesia care team plays an integral role in the line of handover when transferring a patient from the OR to the PACU. During patient transfer, the anesthesiologist is charged with maintaining equipment and monitoring patient vital status in a very busy and hectic environment. Often, patient information handover is received by health care professionals who have little to no prior knowledge of the patient before assuming responsibility for that patient. This brief interaction is a very complex and dynamic bridging between the operating theater and the PACU, and as such, must be closely regulated in order to maintain the same quality of safety standards that are utilized in these two settings.

Hospitals and researchers have made many proposals on how to improve the quality of handovers. ${ }^{10,11}$ One study highlights the implementation of electronic software to affect the accuracy and overall quality of patient handover. ${ }^{12} \mathrm{We}$ propose that a focus on undivided attention has the power to greatly improve postoperative anesthesia handover recall. We underscore the importance of the first handover interaction between the anesthesiologist and the nurse. We observe if the nurses stop whatever they are doing at the moment to look at the doctors when information is passed, or if the nurses continue doing whatever they were doing while passively listening.

We do not acknowledge age-related differences in recall, albeit we are aware they may exist. ${ }^{13}$ This observational study targets undivided attention and attempts to discover whether or not there is a significant difference in outcomes for a patient's safety and recovery when being transferred in an anesthetic setting.

\section{Materials and methods}

This is a case series study of participating subjects. Data were collected in the PACUs of Queen's Hospital and King George's Hospital. These are the two main hospitals of the Barking, Havering and Redbridge National Health Service (NHS) Trust, furnishing 939 and 450 beds, respectively. Both of these modern hospitals were constructed in the past 20 years and serve the same diverse population of over 700,000 residents that fall under the care of the Barking, Havering and Redbridge NHS Trust in the United Kingdom. Participants in this study include nurses and anesthesiologists who play active roles in each postoperative handover of patient information. Subjects were randomly selected from a list of employees scheduled to work in the PACU on days that the data were collected. The participants did not actively volunteer to participate; rather, they agreed to participate only after being selected. Information was recorded and documented regarding the handover of information between multiple anesthesiologists and ten different PACU nurses during the study. There were seven females and three male nurses that participated in the study. While the selection of participants was not congruent with a true randomization, it should be noted that the days on which data were collected were chosen at random and the researchers were unaware of which participants (nurses and anesthesiologists) would be available that day. In addition, participants were not aware of which days were chosen during which to conduct the study, and thus neither the researchers, nor the participants were aware of which surgical cases and patients would be utilized to collect the handover data.

The sample size of 50 handover events was justified based on prior similar studies. The only exclusion criterion regarding participation was refusal by nurses or anesthesiologists to be observed and documented. Data collection of the original handover was instituted immediately at the entrance of patients into their corresponding PACU bay. Twenty minutes after the completion of handover, researchers instituted assessment of recall. Assessment of recall took less than 1 minute in all cases that were included in the dataset. Any and all data that were collected after 21 minutes from the completion of initial handover were not included in the study. The exclusion of these data was done in order to keep our data consistent and to control for any natural decline in memory/recall that might result as time progresses. Information collected by nurses that utilized written notes or the patient chart when asked to recall handover data was not included in the study. There was no control group in this study design. Independent variables of this study design pertain to the number of units handed over during each handover event. Dependent variables include the number of units recalled 20 minutes after the original handover took place. It is expected that some nurses have better recall abilities than others. In order to control for the difference in natural recall abilities and memory capacity, the number of handover events was evenly distributed among each participant. To ensure this, researchers continued to collect data until information regarding five handovers had been received for each nurse.

As each patient entered the PACU, airway management device (rebreathing mask, laryngeal mask airway, or endotracheal tube), bed location, and handover starting time were noted. Patients were also labeled as either an adult or a pediatric case (age $<18$ years). During each handover event, researchers listened to the verbal handover of specific information from the anesthesiologist to the nurse. These specific information points were termed "handover units." 
After the handover was completed, the handover ending time was noted. Handover information/units that were noted include patient name, allergies, operation performed, perioperative analgesics, perioperative antibiotics, postoperative analgesics prescribed, and postoperative antiemetics prescribed. During each handover event, it was also noted whether or not the anesthesiologist had the full, undivided attention of the nurse. A nurse was noted to be giving their undivided attention only if they were not performing any other tasks while the handover was taking place other than listening and asking questions. Tasks that would have precluded the nurse from giving their undivided attention include, but are not limited to, placing electrocardiogram leads, attaching pulse oximeter, attaching nasal cannula, attaching noninvasive blood pressure cuff, administering medications, and writing in patient chart or on scratch paper. Twenty minutes after the original handover ended, the PACU nurse was asked to recall only those handover units that had been verbally relayed to them by the anesthesiologist during the handover. The number of handover events recalled was then logged for each handover event. At the time of recall, the number of patients under the nurse's care was also noted.

\section{Statistical analysis}

The probability of remembering any given handover unit was modeled using a generalized linear mixed model. The model included terms for whether undivided attention was given (yes/no), the nurse receiving the handover, and a residual term. The nurse was treated as a random effect, as the nurses included in the study were considered to represent the wider population of nurses. The number of patients in the nurse's care at the time of handover was not included in the model as it was not possible to model this effectively with the data available. The number of units handed over was excluded from the model as it was not found to be statistically significant. The analysis was performed using $\mathrm{R}$ version 2.15.1 and the lme4 add-on package.

\section{Results}

The data collected is presented in Table 1 and Figures 1-3. Table 1 shows the number of units recalled grouped by whether undivided attention was given and by the total number of units recalled. The percentage of units recalled per handover is shown grouped by whether undivided attention was given (Figure 1), by the total number of units handed over (Figure 2), and by nurse (Figure 3).

Undivided attention was found to increase the odds of remembering any given unit by a ratio of 4.43 (confidence interval 2.03-11.16). When undivided attention is not given, the probability of remembering any given unit is $67.5 \%$. When undivided attention is given, the probability of remembering any given unit is $90.2 \%$.

No significant difference in recall rate is noted between the two hospital sites or between the three airway types. A small difference is seen between adult patients (mean recall rate of $78.9 \%$ ) and pediatric patients (mean recall rate of $75.4 \%$ ); however, this is not statistically significant. A difference is observed depending on the total number of units handed over (see Figure 2); however, this is also not statistically significant.

\section{Discussion}

It can be said with confidence that the presence of undivided attention during the handover of a surgical patient in the PACU has a direct correlation with improved recall of the information discussed during handover. One study showed that having undivided attention while being presented information may not be necessary for simple recognition of the information, but it does have a significant impact on the unassisted recollection of that information at a later time. ${ }^{14}$

Table I Distribution of the number of units recalled, grouped by whether or not undivided attention was given and by the total number of units handed over

\begin{tabular}{|c|c|c|c|c|c|c|c|c|c|}
\hline \multirow{2}{*}{$\begin{array}{l}\text { Undivided attention } \\
\text { given (no/yes) }\end{array}$} & \multirow{2}{*}{$\begin{array}{l}\text { Total units } \\
\text { handed over }\end{array}$} & \multicolumn{8}{|c|}{ Units recalled } \\
\hline & & I & 2 & 3 & 4 & 5 & 6 & 7 & Total \\
\hline \multirow[t]{5}{*}{ No } & 3 & I (I00\%) & 0 & 0 & 0 & 0 & 0 & 0 & I \\
\hline & 4 & 0 & $2(33.3 \%)$ & $2(33.3 \%)$ & $2(33.3 \%)$ & 0 & 0 & 0 & 6 \\
\hline & 5 & 0 & I (14.3\%) & $3(42.9 \%)$ & $2(28.6 \%)$ & I (I4.3\%) & 0 & 0 & 7 \\
\hline & 6 & I (20.0\%) & I (20.0\%) & 0 & $2(40.0 \%)$ & I (20.0\%) & 0 & 0 & 5 \\
\hline & 7 & 0 & 0 & $2(40.0 \%)$ & 0 & I (20.0\%) & I (20.0\%) & I (20.0\%) & 5 \\
\hline \multirow[t]{5}{*}{ Yes } & 3 & 0 & 0 & $3(100 \%)$ & 0 & 0 & 0 & 0 & 3 \\
\hline & 4 & 0 & 0 & I (16.7\%) & $5(83.3 \%)$ & 0 & 0 & 0 & 6 \\
\hline & 5 & 0 & 0 & 0 & $3(50 \%)$ & $3(50 \%)$ & 0 & 0 & 6 \\
\hline & 6 & 0 & 0 & I (14.3\%) & I (14.3\%) & $2(28.6 \%)$ & 3 (42.9\%) & 0 & 7 \\
\hline & 7 & 0 & 0 & 0 & I (25\%) & 0 & I (25\%) & $2(50 \%)$ & 4 \\
\hline
\end{tabular}




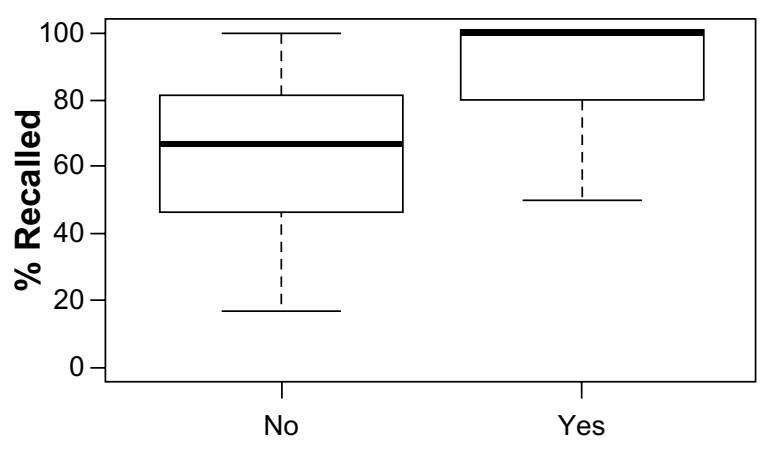

Undivided attention given

Figure I Boxplot of recall rates grouped by whether or not undivided attention was given.

Notes: The thick line indicates the median, the box indicates the interquartile range, and the whiskers indicate the range. When undivided attention was not given, the median recall rate was $66.7 \%$ (with a range of $16.7 \%-100.0 \%$ ), and when undivided attention was given, the median recall rate was $100.0 \%$ (with a range of $50.0 \%-100.0 \%$ ).

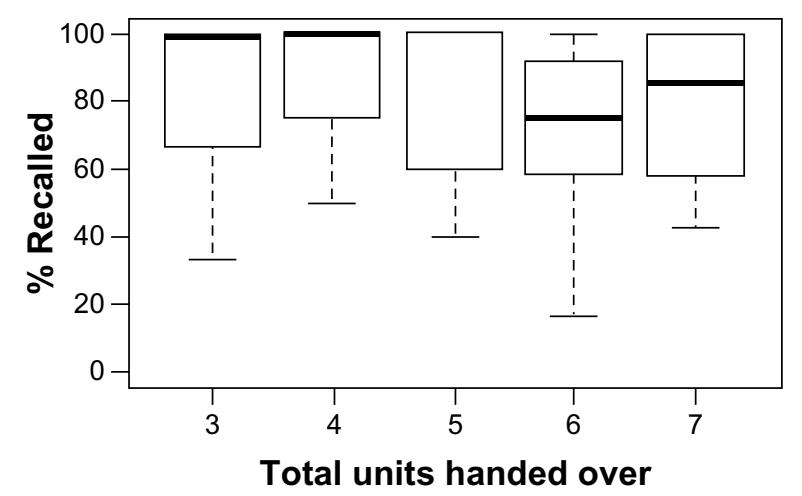

Figure 2 Boxplot of recall rate grouped by total units handed over.

Notes: The thick line indicates the median, the box indicates the interquartile range, and the whiskers indicate the range. The median recall rate was $100.0 \%$ when three units were handed over, $100.0 \%$ when four units were handed over, $80.0 \%$ when five units were handed over, $75.0 \%$ when six units were handed over, and $85.7 \%$ when seven units were handed over.

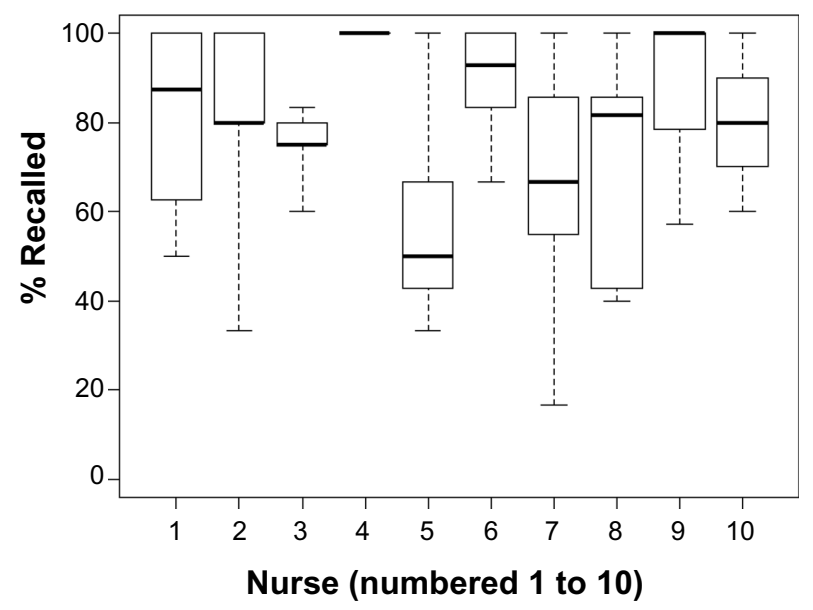

Figure 3 Boxplot of recall rate grouped by nurse.

Note: The thick line indicates the median, the box indicates the interquartile range, and the whiskers indicate the range.
It should be noted that recognition of information was achieved via a provided list of possible correct answer choices, whereas conscious recollection was achieved without any prompting or assistance. ${ }^{14}$ In the stressful and hectic PACU environment, nurses should be able to consciously recall crucial information concerning their patients, as there may be little time to refer to patient charts during an emergency situation. Interruptions during the delivery of health care and the passage of information from one health care provider to another should be minimized so that any negative impact from these interruptions can be avoided. ${ }^{15,16}$

Tape-recorded handovers have been employed in the past, but were utilized in lieu of face-to-face handovers. It has been suggested that tape-recording of the handover should be used as adjunct and in addition to the face-to-face verbal handover to optimize retrieval of information. ${ }^{17}$ It can be understood that information passed via verbal handover is prone to being lost or forgotten due to human error. ${ }^{18} \mathrm{~A}$ proper solution to this problem would be to implement a handover checklist or proforma. In an attempt to improve patient safety standards, many branches of the health care industry have implemented a checklist model into daily patient care. Checklists have been shown to effectively decrease morbidity and mortality by highlighting various aspects of safety, such as improvements in teamwork and communication. ${ }^{19,20}$ Standardized proformas have also been shown to increase overall data transfer up to $20 \%$ when compared to handovers recorded using hand-written notes. ${ }^{18}$ As such, we recommend the implementation of a standardized handover checklist during all postoperative handovers to improve data transfer and communication. The British Medical Association recommends the development of a standardized handover protocol that includes standardized proformas to be utilized during patient handovers. ${ }^{21}$ The Joint Commission on Accreditation of Healthcare Organizations (JCAHO) has recommended the standardization of handover as a National Patient Safety Goal since 2006. ${ }^{22}$ In their model, JCAHO included minimal interruptions as well as verification of proper communication by repeating back information. The implementation of standardized readbacks of information has helped improve safety standards in aviation and air traffic control as well as in the health care setting. ${ }^{23}$ In order to take advantage of the proven benefits of readbacks, we recommend that PACU staff repeat handover information back to anesthesia providers to confirm the accuracy of information as well as to improve information recall.

Various standardized handover mnemonic tools have been created to facilitate information transfer in health care. 
A few examples include IDEAL: identity, diagnosis, events, anticipated, leave; PEDIATRICS: problem list, expected tasks, diagnosis, if/then, administrative data, therapeutics, results, intravenous access, custody, and consents; and DATAS: demographics, assessments, tests, alerts, status. The most widely used handover mnemonic tool in recent use, SBAR, was modeled after naval military procedures and refers to situation, background, assessment, and request. The implementation of SBAR into numerous branches of the health care industry has resulted in improvements in the overall quality and standard of patient safety. ${ }^{24,25}$ As such, we strongly recommend the use of a standardized handover mnemonic tool, such as SBAR, to help regiment the handover process and to take advantage of these improvements in the safety profile of handovers.

Although our case series study was simple and relatively inexpensive, with our data being obtained via firsthand observation of the handover process, we acknowledge the presence of certain limitations. Limitations of the study include those associated with case series studies, including, but not limited to, the Hawthorne effect and measurement bias. In our particular study, the possibility exists that during the study, one or more of the participants (anesthesiologists and nurses) may have become aware that information recall was the variable being assessed. As such, the Hawthorne/ observer effect may have been demonstrated by the nurses' attempts to pay more attention during handover to recall more information when tested by the researchers. In addition, some of the anesthesiologists may have made an effort to include more (or less) information during handover than they normally would had they not been aware of our study on handover information recall.

Another limitation of this study is the possible existence of nonuniform measurement bias. As no reference standard exists for the measurement of undivided attention, our subjective assessment of this variable is susceptible to inaccuracies. Patient handover in the PACU is a complex and widely variable aspect of the postoperative surgical setting. As such, further studies should be done in an effort to standardize the practice of patient handover with the ultimate goal of maximizing patient safety.

Future work in this area may utilize voice recorded and video recorded handovers so that handover data are frozen in media until required. This will provide technology enhanced information storage, and the nurse can access it by rewinding or playing the tapes for recall, eg, to know how much morphine was given, in the OR, what antibiotic was given, and at what time, etc. This would clearly minimize human error. Further studies might expand on the recommendations we have made by testing them directly in the PACU handover setting.

\section{Acknowledgments}

This work was supported by the American University of the Caribbean School of Medicine, Cupecoy, Sint Maarten. The authors thank Ms Sarah Stowell for her help in reviewing this manuscript and for providing statistical assistance.

\section{Author contributions}

All authors contributed toward data analysis, drafting and revising the paper and agree to be accountable for all aspects of the work.

\section{Disclosure}

This manuscript has not been submitted for publication, nor has it been published in whole or in part elsewhere. All authors listed on the title page have read the manuscript and attest to the validity and legitimacy of the data and its interpretation and agree to its submission. The authors report no conflicts of interest in this work.

\section{References}

1. Jeffcott SA, Evans SM, Cameron PA, Chin GS, Ibrahim JE. Improving measurement in clinical handover. Qual Saf Health Care. 2009;18(4): 272-277.

2. Borowitz SM, Waggoner-Fountain LA, Bass EJ, Sledd RM. Adequacy of information transferred at resident sign-out (in-hospital handover of care): a prospective survey. Qual Saf Health Care. 2008;17(1):6-10.

3. Leonard M, Graham S, Bonacum D. The human factor: the critical importance of effective teamwork and communication in providing safe care. Qual Saf Health Care. 2004;13(Suppl 1):i85-i90.

4. Arora V, Johnson J, Lovinger D, Humphrey HJ, Meltzer DO. Communication failures in patient sign-out and suggestions for improvement: a critical incident analysis. Qual Saf Health Care. 2005; 14(6):401-407.

5. Catchpole K, Sellers R, Goldman A, McCulloch P, Hignett S. Patient handovers within the hospital: translating knowledge from motor racing to healthcare. Qual Saf Health Care. 2010;19(4):318-322.

6. Segall N, Bonifacio AS, Schroeder RA, et al; Durham VA Patient Safety Center of Inquiry. Can we make postoperative patient handovers safer? A systematic review of the literature. Anesth Analg. 2012;115(1): 102-115.

7. Shojania KG, Duncan BW, McDonald KM, Wachter RM, Markowitz AJ. Making health care safer: a critical analysis of patient safety practices. Evid Rep Technol Assess (Summ). 2001i-x, 1.

8. Smith AF, Mishra K. Interaction between anaesthetists, their patients, and the anaesthesia team. Br J Anaesth. 2010;105(1):60-68.

9. Smith AF, Pope C, Goodwin D, Mort M. Interprofessional handover and patient safety in anaesthesia: observational study of handovers in the recovery room. Br J Anaesth. 2008;101(3):332-337.

10. Petrovic MA, Martinez EA, Aboumatar H. Implementing a perioperative handoff tool to improve postprocedural patient transfers. Jt Comm J Qual Patient Saf. 2012;38(3):135-142.

11. Boat AC, Spaeth JP. Handoff checklists improve the reliability of patient handoffs in the operating room and postanesthesia care unit. Paediatr Anaesth. 2013;23(7):647-654. 
12. Raptis DA, Fernandes C, Chua W, Boulos PB. Electronic software significantly improves quality of handover in a London teaching hospital. Health Informatics J. 2009;15(3):191-198.

13. Naveh-Benjamin M, Cowan N, Kilb A, Chen Z. Age-related differences in immediate serial recall: dissociating chunk formation and capacity. Mem Cognit. 2007;35(4):724-737.

14. Gardiner JM, Parkin AJ. Attention and recollective experience in recognition memory. Mem Cognit. 1990;18(6):579-583.

15. Brixey JJ, Tang Z, Robinson DJ, et al. Interruptions in a level one trauma center: a case study. Int J Med Inform. 2008;77(4):235-241.

16. Rivera-Rodriguez AJ, Karsh BT. Interruptions and distractions in healthcare: review and reappraisal. Qual Saf Health Care. 2010;19(4): 304-312.

17. O’Connell B, Penney W. Challenging the handover ritual. Recommendations for research and practice. Collegian. 2001;8(3): 14-18.

18. Ferran NA, Metcalfe AJ, O'Doherty D. Standardised proformas improve patient handover: Audit of trauma handover practice. Patient Saf Surg. 2008;2:24

19. Papaconstantinou HT, Jo C, Reznik SI, Smythe WR, Wehbe-Janek H. Implementation of a surgical safety checklist: impact on surgical team perspectives. Ochsner J. 2013;13(3):299-309.
20. Haynes AB, Weiser TG, Berry WR, et al; Safe Surgery Saves Lives Study Group. A surgical safety checklist to reduce morbidity and mortality in a global population. $N$ Engl J Med. 2009;360(5):491-499.

21. Roughton VJ, Severs MP. The junior doctor handover: current practices and future expectations. $J R$ Coll Physicians Lond. 1996;30(3):213-214.

22. [No authors listed]. Joint Commission launches Standards Improvement Initiative. Jt Comm Perspect. 2006;26(10):1.

23. Catchpole KR, Dale TJ, Hirst DG, Smith JP, Giddings TA. A multicenter trial of aviation-style training for surgical teams. J Patient Saf. 2010;6(3):180-186.

24. Beckett CD, Kipnis G. Collaborative communication: integrating SBAR to improve quality/patient safety outcomes. J Healthc Qual. 2009;31(5): 19-28.

25. Haig KM, Sutton S, Whittington J. SBAR: a shared mental model for improving communication between clinicians. Jt Comm J Qual Patient Saf. 2006;32(3):167-175.

\section{Publish your work in this journal}

Advances in Medical Education and Practice is an international, peerreviewed, open access journal that aims to present and publish research on Medical Education covering medical, dental, nursing and allied health care professional education. The journal covers undergraduate education, postgraduate training and continuing medical education including emerging trends and innovative models linking education, research, and health care services. The manuscript management system is completely online and includes a very quick and fair peer-review system. Visit http://www.dovepress.com/testimonials.php to read rea quotes from published authors.

Submit your manuscript here: http://www.dovepress.com/advances-in-medical-education-and-practice-journal 